\title{
CARACTERIZAÇÃO DA LINHA DE COSTA ESTUARINA DA ÁREA URBANA DO MUNICÍPIO DE RIO GRANDE-RS
}

\author{
Thaís Ferreira da Conceição ${ }^{(a)}$, Miguel da Guia Albuquerque(b), Jean Marcel de Almeida \\ Espinoza $^{(c)}$
}

(a) Pós Graduação em Geografia, Universidade Federal do Rio Grande, prof.thaisferreirac@gmail.com

(b) Departamento de Geoprocessamento, Instituto Federal do Rio Grande do Sul, migueldaguia@ gmail.com

(c) Departamento de Geoprocessamento, Instituto Federal do Rio Grande do Sul, jean.espinoza@ riogrande.ifrs.edu.br

\section{Eixo: DINÂMICA E GESTÃO DE ZONAS COSTEIRAS}

\begin{abstract}
Resumo
O presente estudo visa mostrar por meio de imagens de satélite e técnicas de geoprocessamento, o comportamento da linha de costa estuarina do município de Rio Grande, entre a Avenida Major Carlos Pinto e a Rua Saturnino de Brito, nos anos de 2006, 2009, 2012 e 2016. A partir dos dados de linha de costa e da técnica do Polígono de mudança foi possível detectar quais localidades apresentaram um processo de acresção e/ ou erosão. O período entre 2006 e 2009 apresentou as maiores taxas de acresção $(1,78 \mathrm{~m} / \mathrm{ano})$, ao passo que as maiores taxas de erosão foram registradas entre os anos de 2009 a 2012 $(0,85 \mathrm{~m} / \mathrm{ano})$. A partir do exposto tem-se que esse estudo pode vir a contribuir para o município, de forma que o uso dessas ferramentas auxilie na elaboração e aplicação de novas propostas de ordenamento para orla de Rio Grande.
\end{abstract}

Palavras chave: sensores remotos, geoprocessamento, geotecnologias

\section{Introdução}

As zonas costeiras e estuarinas são regiões submetidas a um processo intenso de ocupação, que precisa ser analisado e monitorado para subsidiar os planos de Gestão Pública. Consideradas como áreas peculiares entre dinâmicas sócio-econômicas e elementos naturais, esses ambientes foram às primeiras regiões a serem habitadas tendo em vista a facilidade de obtenção de alimentos através da pesca, questões ligadas a proteção de território e facilidade na movimentação e escoamento de carga (TESSLER \& MAHIQUES, 2007).

Embora grande parte da população mundial esteja alocada nas zonas costeiras, existem poucos estudos que enfoquem os impactos ambientais e desastres naturais relacionados a zona estuarina, decorrentes do processo de uso e ocupação do solo. Tendo em vista que estas localidades sofrem com intensas modificações decorrentes de uma ocupação urbana sem planejamento, o processo de organização do espaço urbano nas regiões costeiras lagunares é de grande interesse para os geógrafos e gestores municipais, pois a partir dele é possível compreender melhor a interação do homem com os sistemas

DOI - 10.20396/sbgfa.v1i2017.2167 - ISBN 978-85-85369-16-3 
naturais (DELMARE et al., 2015).

Para ter uma melhor identificação da suscetibilidade das áreas costeiras e estuarinas, é necessário que se tenha um conhecimento prévio do comportamento da linha de costa. A identificação do grau de suscetibilidade e dos ricos, a delimitação precisa da linha de costa através de feições físicas, e sua modificação através do tempo possibilitam uma análise mais consistente da extensão de eventuais problemas erosivos. Para analisar as modificações históricas na linha de costa, bem como as quantificações da dinâmica urbana, os métodos mais utilizados são as fotografias aéreas e as imagens de satélite (JENSEN, 2009; ALBUQUERQUE, 2013). A partir do exposto, o presente estudo visa caracterizar o comportamento da linha de costa estuarina da área urbana do município de Rio Grande $\mathrm{RS}$, identificando as áreas mais suscetíveis ao processo de erosão e seus impactos nas comunidades estabelecidas nessas localidades.

\section{$1.1 \quad$ Área de Estudo}

O município de Rio Grande (Figura 1) localiza-se no extremo sul do estado do Rio Grande do Sul entre as

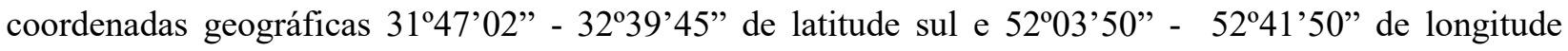
oeste. A localidade está compreendida na planície costeira, sobre uma porção superficial sedimentar quaternária exposta na unidade geomorfológica dessa planície, na sua porção média. A cidade do Rio Grande possui cerca de $2.709 \mathrm{~m}^{2}$ de área, e segundo dados do IBGE (2017) o município tem cerca de 208.641 habitantes. O município é cercado pelas águas do estuário da Lagoa dos Patos, possuindo uma extensa linha de costa estuarina. Para esse estudo foi escolhida a área entre a Avenida Major Carlos Pinto e a Rua Saturnino de Brito, tendo em vista que está região apresentou mudanças significativas relacionadas a questões de aterros e ocupações de suas margens, entre os anos de 2002 a 2016.

\footnotetext{
DOI - 10.20396/sbgfa.v1i2017.2167 - ISBN 978-85-85369-16-3
} 


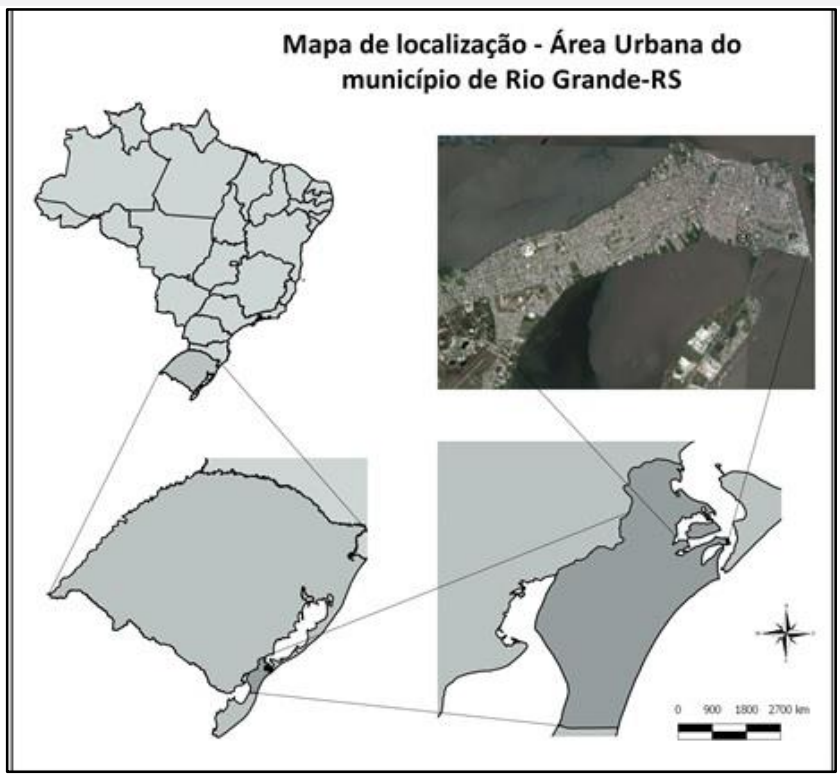

Figura 1 - Mapa de localização da área de estudo.

\section{Materiais e Métodos}

Para desenvolver este estudo, foi necessário criar uma base cartográfica utilizando imagens de satélite do sensor Geoeye dos anos de 2002, 2009, 2012 e 2016. As imagens foram obtidas para as bandas do visível, pancromático e infravermelho próximo. Para o processo de georreferenciamento, e posterior registro do banco de dados, foram obtidos em campo ponto de controle (GCPs) com o auxílio de um GPS Geodésico da marca Leica, operando no modo Stop and Go. Os dados coletados foram referenciados ao datum WGS84, no sistema de projeção UTM (Universal Transverso de Mercator) e zona 22S. A base do GPS foi posicionada no marco do IBGE, situado na Universidade Federal do Rio Grande (FURG), de forma que este proporcionasse as correções dos dados obtidos em tempo real.

Para identificar e monitorar e quantificar a variabilidade da linha de costa estuarina foi utilizado o método do polígono de mudança (ALBUQUERQUE et al., 2013; ANFUSO et al., 2016). Esta metodologia (Figura 2) é utilizada para quantificar a mudança histórica da linha de costa ao longo do tempo, e usa como base duas linhas distintas e vetorizadas, buscando extrair a diferença espacial total entre duas linhas estuarinas vetorizadas. 


\begin{tabular}{|c|c|}
\hline $\begin{array}{l}\text { XVII Simpósio Brasileiro } \\
\text { de Geografia Fisica Aplicada } \\
\end{array}$ & $\begin{array}{l}\text { OS DESAFIOS DA GEOGRAFIA FÍSICA NA FRONTEIRA DO CONHECIMENTO } \\
\text { Instituto de Geociências - Unicamp }\end{array}$ \\
\hline $\begin{array}{l}\text { I Congresso Nacional } \\
\text { de Geografia Física }\end{array}$ & $\begin{array}{c}\text { Campinas - SP } \\
28 \text { de Junho à } 02 \text { de Julho de } 2017\end{array}$ \\
\hline
\end{tabular}

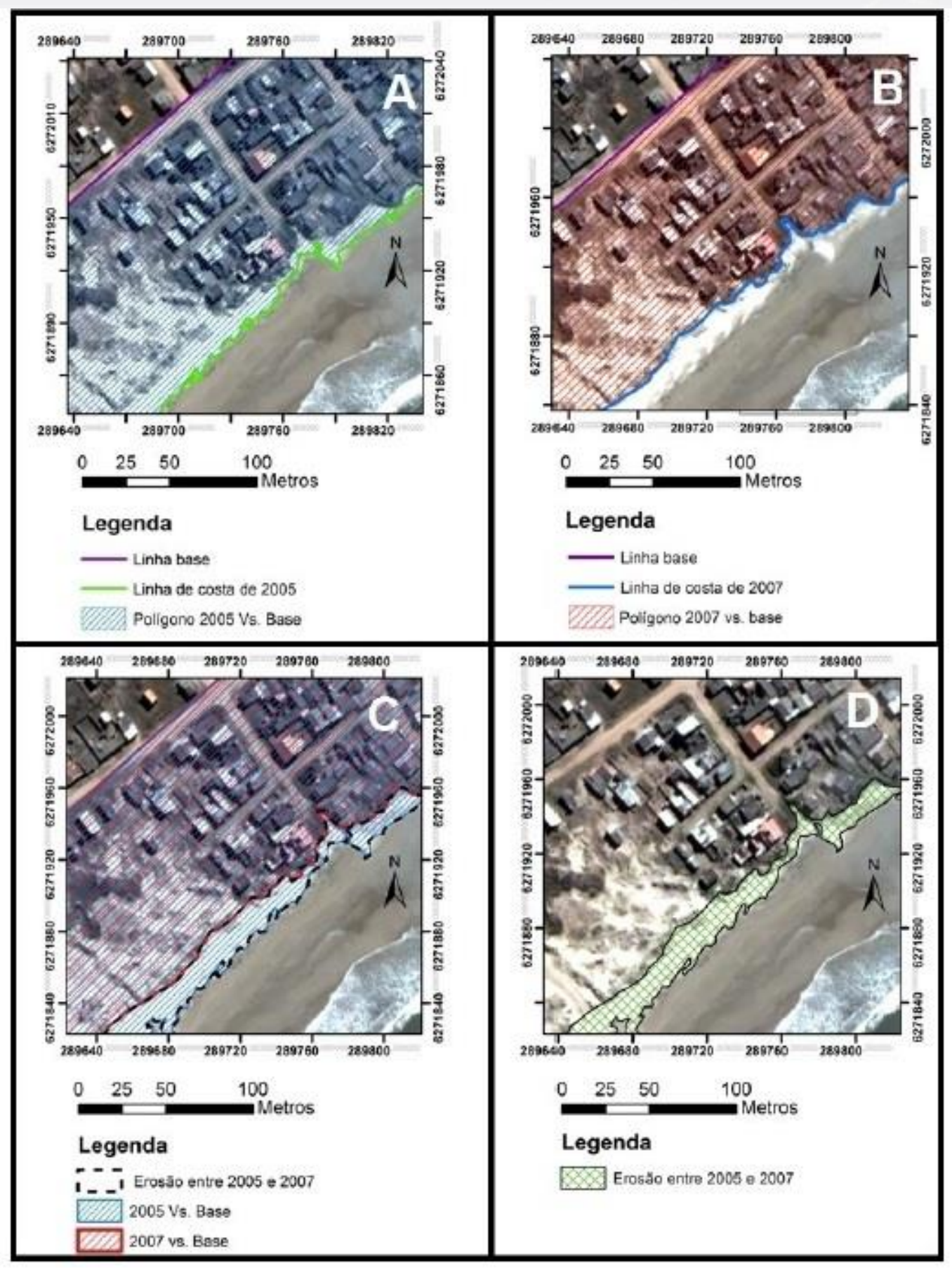

Figura 2 - Caracterização do método do polígono de mudança. Extraído de Albuquerque (2013).

\section{Resultados e Discussões}

O gráfico da Figura 3 mostra a taxa de modificação da costa e o quanto ela sofreu mudanças nos períodos

DOI - 10.20396/sbgfa.v1i2017.2167 - ISBN 978-85-85369-16-3 
entre 2002 a 2016, fazendo um comparativo entre os anos de 2002 a 2009, de 2009 a 2012 e por fim de 2012 a 2016. Foi possível observar que entre os anos de 2006 e 2009 teve-se um acréscimo significante na costa de 1,78 m/ano. Esse acréscimo pode se deve pelas mudanças antrópicas ocorridas na região, tendo como consequência a colocação de aterro e a ocupação sem planejamento da linha de costa estuarina. As taxas de erosão para esse período foram de $0,015 \mathrm{~m} / \mathrm{ano}$.

Entre os anos de 2009 a 2012 e de 2012 a 2016 a linha costeira estuarina apresentou uma tendência erosiva. Os valores de erosão registrados foram de $0,85 \mathrm{~m} / \mathrm{ano}$ e $0,69 \mathrm{~m} / \mathrm{ano}$, respectivamente. A erosão observada para esse período pode ser atribuída à ausência de vegetação ciliar nas margens da orla de Rio Grande, o que impossibilita a estabilidade dos sedimentos e contribui para a remoção dos mesmos durante a passagem de eventos extremos na região. Para a erosão ocorrida no intervalo entre 2012 a 2016, a retração pode estar associada, também, a passagem de um ciclone no dia 26 de outubro de 2016, onde foram registrados ventos de até $80 \mathrm{~km} / \mathrm{h}$, e uma elevação do nível da lagoa na área estudada.

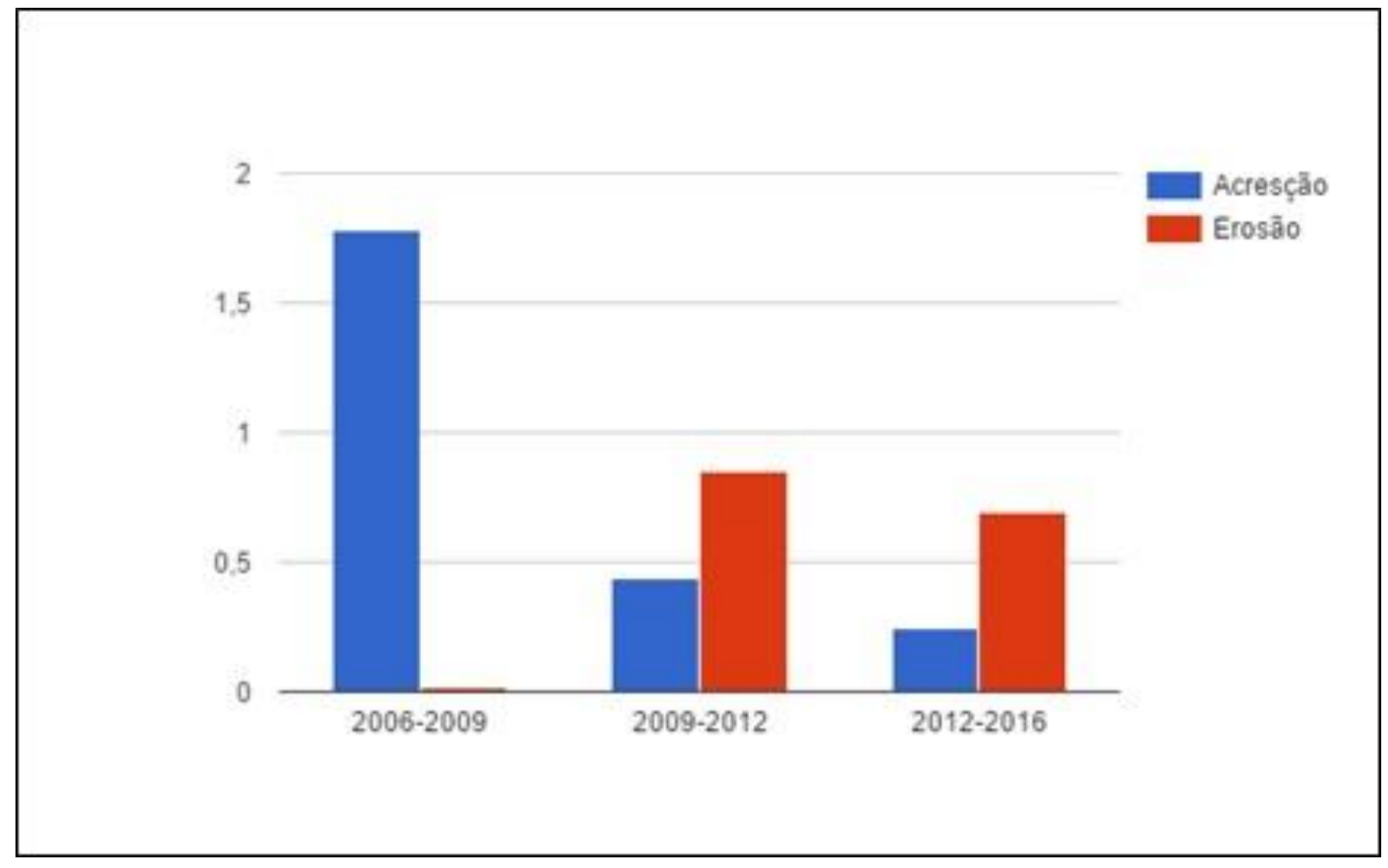

Figura 3 - Gráfico da taxa de modificação da costa

\section{Conclusões}

A aplicação do método de detecção de mudança se mostrou eficiente na identificação das mudanças ocorridas ao longo da costa estuarina do município de Rio Grande, mostrando a variabilidade durante o período de 2006 a 2016, decorrentes de aterramentos feitos pela Prefeitura do município ou pelos

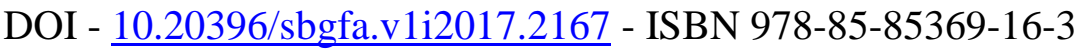


processos de ocupação indevida em algumas localidades. Os valores de acresção foram atribuídos a essas intervenções na orla. Já as taxas de erosão encontradas entre 2009 e 2012 e 2012 a 2016 podem ser atribuídas à falta de uma vegetação ciliar, a qual seria responsável pela estabilidade da região, e a questões relacionadas a eventos meteorológicos de grande impacto (i.e. o evento ocorrido em 26 e 27 de outubro de 2016).

A partir do exposto tem-se que esse estudo pode vir a contribuir para a administração pública do município, por meio do mapeamento urbano da orla de Rio Grande. Nesse sentido, espera-se que os resultados elaborados com o uso dessas ferramentas do geoprocessamento auxiliem na elaboração e aplicação de novas propostas que possam beneficiar a cidade e a população, proporcionando aos moradores uma melhor qualidade de vida.

\section{REFERÊNCIAS}

ALBUQUERQUE, M.G.; ESPINOZA, J.; TEIXEIRA, P.; DE OLIVEIRA, A.; CORREA, I.; CALLIARI, L. Erosion or coastal variability: an evaluation of the DSAS and change polygon methods for the determination of erosive process on sandy beaches - Journal of Coastal Research, SI(65): 1710-1714 (2013)

ALBUQUERQUE, M. G. Análise espaço-temporal das causas da variabilidade da linha de costa e erosão na praia do Hermenegildo, RS. Porto Alegre: Universidade Federal do Rio Grande do Sul - UFRGS. Tese do Programa de Pós-Graduação em Geociências. 127 f. (2013)

ANFUSO, G.; BOWMAN, D.; DANESE, C.; PRANZINI, E. - Transect based analysis versus area based analysis to quantify shoreline displacement: spatial resolutions issues - Environmental Monitoring Assessment, 188 : 568 (2016)

DELAMARE, T.O; SIMON, A. L. H.; SATO, S.E. - Dinâmica de uso da terra e alterações na linha de costa lagunar: estudo em uma Colônia de Pescadores de Pelotas, RS, Brasil - Revista de Gestão Costeira Integrada / Journal of Integrated Coastal Zone Management, 15(2):237-248 (2015)

IBGE - Instituto Brasileiro de Geografia e Estatística. IBGE CIDADES@, 2017. Disponível em: < http://cidades.ibge.gov.br/xtras/perfil.php?lang=\&codmun=431560\&search=rio-grande-do-sul|rio-grande $>\quad$ Acesso em: Janeiro. 2017.

JENSEN, J. R. Sensoriamento remoto do ambiente: uma perspectiva em recursos terrestres. São José dos Campos: Parêntese. p. 129-150. (2009)

SMITH, M.J. \& CROMLEY, R.G. - Mensure historical coastal change using GIS and the change polygon approach. Transactions in GIS, 16(1): 3-15

TESSLER, M.G., MAHIQUES, M.M. - Processos oceânicos e a fisiografia dos fundos marinhos. In: TEIXEIRA, W.; TOLEDO, M.C.M. DE; FAIRCHILD, T. R.; TAIOLI, F. (coord.), Decifrando a Terra. São Paulo: Editora Oficina de Textos, 2000. pp.262-284 